\title{
GLOBAL EXISTENCE AND UNIQUENESS OF THE WEAK SOLUTION IN THIXOTROPIC MODEL
}

\author{
AMIRA RAHAI, AMAR GUESMIA* \\ Laboratory of Applied Mathematics and History and Didactics of Mathematics (LAMAHIS), Department of \\ Mathematics, University 20 august 1955 Skikda, Algeria \\ *Corresponding author: guesmiaamar19@gmail.com

\begin{abstract}
In this paper, we study global existence, uniqueness and boundedness of the weak solution for the system $(P)$ which is formulated by two subsystems $\left(P_{1}\right)$ and $\left(P_{2}\right)$, the first describes the thixotropic problem and the second describes the diffusion degradation of $c$, using Galerkin's method, Lax-Milgran's and maximum principle. Moreover we show that the unique solution is positive.
\end{abstract}

\section{INTRODUCTION}

The phenomenon of thixotropy has recently attracted a great deal of attention. The term was first applied [3] to an "isothermal reversible sol-gel transformation". As the gel state is often merely one of high viscosity, the definition has been made more general, and the term is then applied [5] to any " isothermal reversible decrease of viscosity with increase of rate of shear".

Colloidal solutions provide the more common examples of thixotropy and may be divided into three important classes :

- Solutions in Newtonian liquids of lyophilic substances whose molecules are of great length, e.g., gelatine, starch and many synthetic polymers.

- Suspensions of solid particles such as pigments in oils, or clays in water.

Received November $10^{\text {th }}, 2020$; accepted December $7^{\text {th }}, 2020$; published February $1^{\text {st }}, 2021$.

2010 Mathematics Subject Classification. Primary 90C57, 90C59 Secondary 90C49.

Key words and phrases. thixotropic; global solution; boundedness; positive solution.

(C)2021 Authors retain the copyrights of their papers, and all open access articles are distributed under the terms of the Creative Commons Attribution License. 
- Concentrated emulsions ( [11], [12]) of oil droplets in water; foams of gas bubbles in water (with, of course, stabilising agents).

Thixotropic fluids are used widely in civil engineering, food, cosmetic as well as pharmaceutical industries, and impact every aspect of our lives. As emulsions, suspensions, or polymeric gels, they are very different from each other compositionally, but most of them have one thing in common, i.e., the existence of microstructures. The microstructures are changeable and may comprise a network of flocculated colloidal particles, tangles of polymers, or a spatial arrangement of suspended particles or drops [1].

Thixotropic fluids have a lot of special characters, such as aging, rejuvenation, and viscosity bifurcation [14] and by rate dependent properties associated to their structural level. The behavior of these substances under rheological tests have been analyzed in many scientific works ( [2], [9], [10], [13], [15]), which was firstly proposed by Moore [8] in 1959. All of these scientific works were presented a qualitative explanation of the break down and build-up processes of the structure.

In this paper, we are interested in the study of the global existence and uniqueness of weak positive solution for the elliptic-parabolic model's.

Our model is defined as follows:

$$
(P)\left\{\begin{array}{l}
\left(P_{1}\right) \begin{cases}u_{t}+\Delta u-\lambda \operatorname{div}\left[u \frac{\nabla\left(c-u_{0}\right)}{\sqrt{\beta+\left|\nabla\left(c-u_{0}\right)\right|^{2}}}\right]+u=u_{0} & (t, x) \in \mathbb{R}^{+} \times \Omega \\
u=0 & \partial \Omega \\
u(0, x)=u_{0} & \left(P_{2}\right) \begin{cases}-\Delta c+\tau c=0 & x \in \Omega \\
c=g & \partial \Omega\end{cases} \end{cases}
\end{array}\right.
$$

Where $u(t, x)$ is a function denotes the speed of fluid in the position $x \in \Omega \subset \mathbb{R}^{2}$ or $\mathbb{R}^{3}, \Omega$ is a bounded convex domain with smooth boundary $\partial \Omega \in H^{\frac{3}{2}}(\partial \Omega), \lambda>0$ is the viscosity of the fluid, $\beta>0$ is a parameter constant, $c$ denotes the concentration of chemical signal that stimulates the fluid. The parameter $\tau$ is a time constant and it is expressed on the one hand the movement of fluid and secondly the diffusion degradation of $c$.

To simplify the solution of the system $(P)$, a decomposition of $(P)$ into two subsystem $\left(P_{1}\right)$ and $\left(P_{2}\right)$ are adopted. Galerkin's method is very important to help us to demonstrate the existence and uniqueness of a weak solution for system $\left(P_{1}\right)$. To prove the existence and uniqueness of a weak solution for system $\left(P_{2}\right)$, we use Lax-Milgram's theorem and maximum principle. However this theorem can not be applied directly because it is nonhomogenous system. For this reason an adoptation of Trace theorem it used to simplify the $\operatorname{system}\left(P_{2}\right)$. Therefore we have the existence and uniqueness of a weak solution for system $(P)$. Moreover we show that the solution is positive. 
The following initial-boundary conditions on $u_{0}$ and $g$ assumptions are used to prove the proposed solution of $(P)$

- $H_{1}: g \in L^{\frac{1}{2}}(\partial \Omega)$.

- $H_{2}: g \in L^{\frac{3}{2}}(\partial \Omega)$.

- $H_{3}: u_{0} \in L^{2}(\Omega)$.

- $H_{4}: u_{0} \geq 0$ and $g \geq 0$.

If the hypothesis $H_{1}$ is satisfies and using the theorem of trace, one can find a lifting of this trace which we denote $R(g) \in H_{0}^{1}(\Omega)$. Thus by definition it verefies $\gamma_{0}(R(g))=g$. Now we looking for $c$ having the form $c=\widetilde{c}+R(g)$ reduves the problem $\left(P_{2}\right)$ to $\widetilde{c}$.

$$
\left(\widetilde{P_{2}}\right) \begin{cases}-\Delta \widetilde{c}+\tau \widetilde{c}-\Delta R(g)+\tau R(g)=0 & x \in \Omega \\ \widetilde{c}=0 & \text { on } \partial \Omega\end{cases}
$$

Definition 1.1. We say $(u, \widetilde{c}) \in L^{2}\left(0, T, H_{0}^{1}(\Omega)\right) \times H_{0}^{1}(\Omega)$ with $u_{t} \in L^{2}\left(0, T, H^{-1}(\Omega)\right)$ is a weak solution of the problem $(P)$ if and only if

$$
\left\langle u_{t}, v\right\rangle+B(u, v, t)=\left(u_{0}, v\right)
$$

where

$$
a(\widetilde{c}, q, t)=l(q)
$$

\section{where}

$$
\left\{\begin{array}{c}
B(u, v, t)=-\int_{\Omega} \nabla u \nabla v d x+\int_{\Omega}(\delta \tau c+1) u v d x-\delta \int_{\Omega} u \nabla u_{0} \nabla v d x \\
a(\widetilde{c}, q, t)=\int_{\Omega}(\nabla \widetilde{c} \nabla q+\tau \widetilde{c} q) d x \\
l(q)=-\int_{\Omega}(\nabla R(g) \nabla q+\tau R(g) q) d x
\end{array}\right.
$$

for $\operatorname{all}(v, q) \in\left(H_{0}^{1}(\Omega)\right)^{2}, 0 \leq t \leq T$,

$$
u(0, x)=u_{0} \in L^{2}(\Omega)
$$

and

$$
\delta=\frac{\lambda}{\sqrt{\beta+\left|\nabla\left(c-u_{0}\right)\right|^{2}}}
$$

Remark 1.1. Note that $u \in C\left([0, T], L^{2}(\Omega)\right)$ as $u \in L^{2}\left(0, T, H_{0}^{1}(\Omega)\right)$ and $u_{t} \in L^{2}\left(0, T, H^{-1}(\Omega)\right)$. Then equation 1.3 makes sense. 


\section{Existence of Weak solution of the Problem $(P)$}

In this section, we are interested in the study of the existence and uniqueness of weak solution of the problem $\left(P_{1}\right)$, which its variational formulat is given by equation 1.1 using Galerkin's method and use the theorem of Lax-Milgram to study the existence and uniqueness of weak solution of the problem $\left(P_{2}\right)$, which its variational formulat is given by equation 1.2. So we have the existence and uniqueness of weak solution of the problem $(P)$.

\subsection{Existence of weak solution of the problem $\left(P_{2}\right)$.}

Theorem 2.1. If the hypothesis $H_{1}$ holds. Then the problem $\left(P_{2}\right)$ has only one solution $c \in H^{1}(\Omega)$ for any $q \in H^{1}(\Omega)$.

By applying the theorem of Lax-Milgram, the solution $\widetilde{c}$ of the problem 1.2 exists and it is unique. So $\left(P_{2}\right)$ has unique solution.

Remark 2.1. Elliptic regularity theorem remains valid provided that the boundary condition $g$ is in the space $L^{\frac{3}{2}}(\partial \Omega)$ which is the image by the operator trace space $H^{2}(\Omega)$.

Remark 2.2. [17] If $c \in H^{2}(\Omega)$ and ( $c$ is a solution of problem $\left(P_{2}\right)$ ) this implies that $c \in W^{1, q}(\Omega)($ $H^{2}(\Omega) \hookrightarrow W^{1, q}(\Omega)$ for $\left.1 \leq q \leq 2\right)$.

Using the Maximum Principle one can show that the solution of the problem $\left(P_{2}\right)$ is positive as follows. Multiplying the first equation of $\left(P_{2}\right)$ by $q \in H_{0}^{1}(\Omega)$, we obtain other variational formulat for problem $\left(P_{2}\right)$

$$
\left(\widetilde{P_{3}}\right) \int_{\Omega}(\nabla c \nabla q+\tau c q) d x=0
$$

Proposition 2.1. [16] If $g \in L^{\frac{3}{2}}(\partial \Omega)$ and $c \in H^{1}(\Omega) \cap C(\bar{\Omega})$ then the problem $\left(\widetilde{P_{3}}\right)$ have a positive solution $c$.

Proof. As $\partial \Omega$ is smooth enough and $g \in L^{\frac{3}{2}}(\partial \Omega)$ then $c \in H^{2}(\Omega)$. And as $\Omega \subset \mathbb{R}^{2}$ or $\mathbb{R}^{3}$, by embedding of Sobolev spaces $\left(H^{2}(\bar{\Omega}) \hookrightarrow C(\bar{\Omega})\right)$ this implies that $c \in C(\bar{\Omega})$. If $c=g \geq 0$ on $\partial \Omega$, then $c^{-}=\min (c, 0) \in$ $H_{0}^{1}(\Omega)$. So, we have

$$
\begin{gathered}
\int_{\Omega} c c^{-} d x=\int_{\Omega}\left(c^{-}\right)^{2} d x \\
\int_{\Omega} \nabla c \nabla c^{-} d x=\int_{\Omega}\left(\nabla c^{-}\right)^{2} d x,
\end{gathered}
$$

Since the support of functions $c^{-}$and $c^{+}=\max (c, 0)$ is set $A(x)=\{x / u(x)=0\}$.

This implies that $\nabla u=0$ on $A(x)$. As $c=c^{+}+c^{-}$, thus we have 


$$
0=\int\left(\left(\nabla c^{-}\right)^{2}+\tau\left(c^{-}\right)^{2}\right) d x \geq \min (1, \tau)\left\|c^{-}\right\|_{H_{0}^{1}(\Omega)}^{2}
$$

Finally, we find $c^{-}=0$.

\subsection{Existence of weak solution of the problem $\left(P_{1}\right)$.}

Lemma 2.1. i) For all $v \in H_{0}^{1}(\Omega)$ then $B(., ., t)$ is continuous in $H_{0}^{1}(\Omega) \times H_{0}^{1}(\Omega)$, there exists a constant positive $M$ such that

$$
|B(u, v, t)| \leq M\|u\|_{H^{1}(\Omega)}\|v\|_{H^{1}(\Omega)}
$$

ii) For any $u \in H_{0}^{1}(\Omega)$ and $H_{2}$ is hold. Then exists a constant positive $\alpha$ such that

$$
B(u, u, t) \geq \alpha\|u\|_{H_{0}^{1}(\Omega)}^{2}
$$

Proof. i) We use the Cauchy-Shwartz inequality and $C \in H^{1}(\Omega) \hookrightarrow L^{q}(\Omega)$ for any $q \in\left[1, \frac{2 n}{n-2}[\right.$ with $n=2$ or $n=3$, we obtain i) as follows

$$
\begin{aligned}
|B(u, v, t)| & \leq\|\nabla u\|_{L^{2}(\Omega)}\|\nabla v\|_{L^{2}(\Omega)}+\left[|\delta \tau|\|c\|_{L^{2}(\Omega)}+1\right]\|u\|_{L^{2}(\Omega)}\|v\|_{L^{2}(\Omega)} \\
& +|\delta|\|u\|_{L^{2}(\Omega)}\left\|u_{0}\right\|_{L^{2}(\Omega)}\|\nabla v\|_{L^{2}(\Omega)} \\
& \leq M\|u\|_{H^{1}(\Omega)}\|v\|_{H^{1}(\Omega)} .
\end{aligned}
$$

ii) Making use of $-\Delta c+\tau c=0$ the expression of $B(u, u, t)$ becomes

$$
\begin{aligned}
B(u, u, t) & =-\int(\nabla u)^{2} d x+\int(\delta \tau c+1) u^{2} d x-\delta \int u \nabla u \nabla u_{0} d x \\
& =-\int(\nabla u)^{2} d x+\int(\delta \tau c+1) u^{2} d x-\frac{\delta}{2} \int(\nabla u)^{2} \nabla u_{0} d x \\
& =\int\left(-1-\frac{\delta}{2} \nabla u_{0}\right)(\nabla u)^{2} d x+\int(\delta \tau c+1) u^{2} d x \\
& \geq\|\nabla u\|_{L^{2}(\Omega)}^{2} .
\end{aligned}
$$

Finally, by Poincarre inequality yields,

$$
B(u, u, t) \geq \alpha\|u\|_{H_{0}^{1}(\Omega)}^{2}
$$

2.2.1. Galerkin approximations. To demonstrate the existence of weak solution of the problem $\left(P_{1}\right)$ via the method of Galerkin, we assume $w_{k}=w_{k}(x)$ are smooth function verifying

$$
\left\{w_{k}\right\}_{k=1}^{\infty} \text { is an arthogonal basis of } H_{0}^{1}(\Omega)
$$

and

$$
\left\{w_{k}\right\}_{k=1}^{\infty} \text { is an arthonormal basis of } L^{2}(\Omega) \text {. }
$$


Consider a positive integer $m$. We will look for a function $u_{m}:[0, T] \rightarrow H_{0}^{1}(\Omega)$ of the form

$$
u_{m}:=\sum_{k=1}^{m} d_{m}^{k}(t) w_{k}
$$

which satisfies

$$
d_{m}^{k}(0)=\left(u_{0}, w_{k}\right)
$$

and

$$
\left\langle u_{m}^{\prime}, w_{k}\right\rangle+B\left(u_{m}, w_{k}, t\right)=\left(u_{0}, w_{k}\right), \quad 0 \leq t \leq T \quad \text { and } \quad k=1, \ldots, m
$$

where $u^{\prime}=u_{t}$ and here $(.,$.$) denotes the scalar product in L^{2}(\Omega)$.

Theorem 2.2. (construction of the approximate solution) For each integer $m$, there exists a unique function $u_{m}$ of the form equation 2.5 satisfying equation 2.6 and equation 2.7 .

Proof. Assuming $u_{m}$ has the structure equation 2.5. Substituting equation 2.5 into equation 2.7 and using equation 2.4 we obtained

$$
d_{m}^{\prime k}(t)+\sum_{l=1}^{m} d_{m}^{l} B\left(w_{l}, w_{k}, t\right)=d_{m}^{k}(0), \quad 0 \leq t \leq T \quad \text { and } \quad k=1, \ldots, m
$$

According to standard existence theory for ordinary differential equations, there exists a unique absolutely continuous functions $d_{m}(t)=\left(d_{m}^{1}, d_{m}^{2}, \ldots, d_{m}^{m},\right)$ satisfying equation 2.6 and equation 2.8. So $u_{m}$ of the form equation 2.5 satisfies equation 2.6 and equation 2.7 for all $t \in[0, T]$.

2.2.2. Energy estimates. We propose now to send $m$ to infinity and show a subsequence of our solutions $u_{m}$ of the approximation problems equation 2.6 and equation 2.7 converges to a weak solution of $\left(P_{1}\right)$. For this we will need some uniform estimates.

Theorem 2.3. ( Energy estimates ) [17]. There exists a constant $C$, depending only on $\Omega, T$ and $c$, such that

$$
\max _{0 \leq t \leq T}\left\|u_{m}\right\|_{L^{2}(\Omega)}+\left\|u_{m}\right\|_{L^{2}\left(0, T, H_{0}^{1}(\Omega)\right)}+\left\|u_{m}^{\prime}\right\|_{L^{2}\left(0, T, H^{-1}(\Omega)\right)} \leq C\left\|u_{0}\right\|_{L^{2}(\Omega)} \quad \text { for } \quad m=1,2, \ldots
$$

Proof. (1) Multiplying equation 2.7 by $d_{m}^{k}(t)$, summing for $k=1, \ldots, m$, and then recalling equation 2.5 we find

$$
\left(u_{m}^{\prime}, u_{m}\right)+B\left(u_{m}, u_{m}, t\right)=\left(u_{0}, u_{m}\right)
$$


for all $0 \leq t \leq T$. From Lemme 2.1, there exists constant $\alpha>0$ such that

$$
\alpha\left\|u_{m}\right\|_{H_{0}^{1}(\Omega)}^{2} \leq B\left(u_{m}, u_{m}, t\right)
$$

for all $0 \leq t \leq T, m=1, \ldots$ Furthermore $\left|\left(u_{0}, u_{m}\right)\right| \leq \frac{1}{2}\left\|u_{0}\right\|_{L^{2}(\Omega)}^{2}+\frac{1}{2}\left\|u_{m}\right\|_{L^{2}(\Omega)}^{2}$, and $\left(u_{m}^{\prime}, u_{m}\right)=$ $\frac{d}{d t}\left(\left\|u_{m}\right\|_{L^{2}(\Omega)}^{2}\right)$ for a.e. $0 \leq t \leq T$. Consequently equation 2.10 yields the inequality

$$
\frac{d}{d t}\left(\left\|u_{m}\right\|_{L^{2}(\Omega)}^{2}\right)+2 \alpha\left\|u_{m}\right\|_{H_{0}^{1}(\Omega)}^{2} \leq C_{1}\left\|u_{m}\right\|_{L^{2}(\Omega)}^{2}+C_{2}\left\|u_{0}\right\|_{L^{2}(\Omega)}^{2}
$$

for all $0 \leq t \leq T$ and appropriate constants $C_{1}$ and $C_{2}$.

(2) Now write

and

$$
\varphi(t):=\left\|u_{m}\right\|_{L^{2}(\Omega)}^{2}
$$

$$
\zeta(t):=\left\|u_{0}\right\|_{L^{2}(\Omega)}^{2} .
$$

Then equation 2.12 implies

$$
\varphi(t) \leq e^{C_{1} t}\left(\varphi(0)+C_{2} \int_{0}^{T} \zeta(s) d s\right)(0 \leq t \leq T)
$$

Since $\varphi(0)=\left\|u_{m}(0)\right\|_{L^{2}(\Omega)}^{2} \leq\left\|u_{0}\right\|_{L^{2}(\Omega)}^{2}$ by equation 2.6, we obtain from equations $2.13-2.16$ the estimate

$$
\max _{0 \leq t \leq T}\left\|u_{m}\right\|_{L^{2}(\Omega)} \leq C\left\|u_{0}\right\|_{L^{2}(\Omega)}
$$

(3) Integrate inequality equation 2.12 from 0 to $T$ and we employ the inequality equation 2.17 to find

$$
\left\|u_{m}\right\|_{L^{2}\left(0, T, H_{0}^{1}(\Omega)\right)}^{2}=\int_{0}^{T}\left\|u_{m}\right\|_{H_{0}^{1}(\Omega)}^{2} d t \leq C\left\|u_{0}\right\|_{L^{2}(\Omega)}^{2} .
$$


(4) Fix any $v \in H_{0}^{1}(\Omega)$, with $\|v\|_{H_{0}^{1}(\Omega)}^{2} \leq 1$, and write $v=v^{1}+v^{2}$, where $v^{1} \in \operatorname{span}\left(w_{k}\right)_{k=1}^{k=m}$, and $\left(v^{2}, w_{k}\right)=0(k=1, \ldots, m)$. We use equation 2.7 , we deduce for all $0 \leq t \leq T$ that

$$
\left(u_{m}^{\prime}, v^{1}\right)+B\left(u_{m}, v^{1}, t\right)=\left(u_{0}, v^{1}\right)
$$

then equation 2.5 implies

$$
\left\langle u_{m}^{\prime}, v\right\rangle=\left(u_{m}^{\prime}, v\right)=\left(u_{m}^{\prime}, v^{1}\right)=\left(u_{0}, v^{1}\right)-B\left(u_{m}, v^{1}, t\right)
$$

consequently

$$
\left|\left\langle u_{m}^{\prime}, v\right\rangle\right| \leq C\left(\left\|u_{0}\right\|_{L^{2}(\Omega)}^{2}+\left\|u_{m}\right\|_{H_{0}^{1}(\Omega)}\right) .
$$

Simce $\left\|v^{1}\right\|_{H_{0}^{1}(\Omega)}^{2} \leq\|v\|_{H_{0}^{1}(\Omega)}^{2} \leq 1$. Thus

$$
\left\|u_{m}^{\prime}\right\|_{H^{-1}(\Omega)} \leq C\left(\left\|u_{0}\right\|_{L^{2}(\Omega)}^{2}+\left\|u_{m}\right\|_{H_{0}^{1}(\Omega)}\right)
$$

and therefore

$$
\left\|u_{m}^{\prime}\right\|_{L^{2}\left(0, T, H^{-1}(\Omega)\right)}^{2}=\int_{0}^{T}\left\|u_{m}^{\prime}\right\|_{H^{-1}(\Omega)}^{2} d t \leq C \int_{0}^{T}\left(\left\|u_{0}\right\|_{L^{2}(\Omega)}^{2}+\left\|u_{m}\right\|_{H_{0}^{1}(\Omega)}\right) d t \leq C\left\|u_{0}\right\|_{L^{2}(\Omega)} .
$$

2.2.3. Existence and uniqueness. Next we pass to limit as $m \rightarrow \infty$, to build a weak solution of our initial boundary-value problem $\left(P_{1}\right)$.

Theorem 2.4. (Existence of weak solution). Under hypothesis $H_{2}$ and $H_{3}$, there exists a weak solution of $\left(P_{1}\right)$.

Proof. (1) According to the energy estimates equation 2.9, we see that the sequence $\left\{u_{m}\right\}_{m=1}^{\infty}$ is bounded in $L^{2}\left(0, T, H_{0}^{1}(\Omega)\right)$ and $\left\{u_{m}^{\prime}\right\}_{m=1}^{\infty}$ is bounded in $L^{2}\left(0, T, H^{-1}(\Omega)\right)$. Consequently there exists a subsequence which is also noted by $\left\{u_{m}\right\}_{m=1}^{\infty}$ and a function $u \in L^{2}\left(0, T, H_{0}^{1}(\Omega)\right)$, with $u^{\prime} \in$ $L^{2}\left(0, T, H^{-1}(\Omega)\right)$, such that

$$
\begin{array}{llll}
u_{m} \rightarrow u & \text { weakly in } & L^{2}\left(0, T, H_{0}^{1}(\Omega)\right) \\
u_{m}^{\prime} \rightarrow u^{\prime} & \text { weakly in } & L^{2}\left(0, T, H^{-1}(\Omega)\right) .
\end{array}
$$


(2) Next fix an integer $N$ and choose a function $v \in C^{1}\left(0, T, H_{0}^{1}(\Omega)\right)$ having the form

$$
v(t)=\sum_{k=1}^{N} d^{k}(t) w_{k}
$$

where $\left\{d^{k}\right\}_{k=1}^{N}$ are given smooth functions. We choose $m \geq N$, multiply equation 2.7 by $d^{k}(t)$, sum for $k=1, \ldots, N$, and then integrate with respect to $t$ to find

$$
\left\langle u^{\prime}, v\right\rangle+B(u, v, t)=\left(u_{0}, v\right) \quad \forall v \in H_{0}^{1}(\Omega) \quad \text { and } \forall t \in[0, T]
$$

and from remark 1.1 we have $u \in C\left(0, T, L^{2}(\Omega)\right)$.

(3) In order to prove $u(0)=u_{0}$, we first note from equation 2.21 that

$$
\int_{0}^{T}-\left\langle u, v^{\prime}\right\rangle+B(u, v, t) d t=\int_{0}^{T}\left(u_{0}, v\right) d t+(u(0), v(0))
$$

for each $v \in C^{1}\left(0, T, H_{0}^{1}(\Omega)\right)$ with $v(t)=0$. Similary, from equation 2.20 we deduce

$$
\int_{0}^{T}-\left\langle u_{m}, v^{\prime}\right\rangle+B\left(u_{m}, v, t\right) d t=\int_{0}^{T}\left(u_{0}, v\right) d t+\left(u_{m}(0), v(0)\right)
$$

we use again equation 2.18 , we obtain

$$
\int_{0}^{T}-\left\langle u, v^{\prime}\right\rangle+B(u, v, t) d t=\int_{0}^{T}\left(u_{0}, v\right) d t+\left(u_{0}, v(0)\right)
$$

since $u_{m}(0) \rightarrow u_{0}$ in $L^{2}(\Omega)$. Comparing equation 2.23 and equation 2.25, we conclude $u(0)=u_{0}$.

Theorem 2.5. (Uniqueness of a weak solutions) A weak solution of $\left(P_{1}\right)$ is unique. 
Proof. We suppose there exists two weak solutions $u_{1}$ and $u_{2}$. We put

$$
U=u_{2}-u_{1}
$$

then $U$ is also a solution of $\left(P_{1}\right)$ with $U_{0}=\left(u_{2}-u_{1}\right)(0) \equiv 0$. Setting $v=U$ in identity equation 2.19 we have

$$
\frac{d}{d t}\left(\frac{1}{2}\|U\|_{L^{2}(U)}^{2}+B(U, U, t)\right)=0
$$

From Lemma 2.1 we have $B(U, U, t) \geq \alpha\|U\|_{H_{0}^{1}(U)}^{2} \geq 0$, so $\frac{d}{d t}\left(\frac{1}{2}\|U\|_{L^{2}(U)}^{2}\right) \leq 0$, then integrate with respect to $t$ to find

$$
\|U\|_{L^{2}(U)}^{2} \leq\left\|U_{0}\right\|_{L^{2}(U)}^{2}=0
$$

thus $U \equiv 0$.

2.3. Global solution of problem $(P)$. Our main results in this paper are stated as follows.

Theorem 2.6. i) if $c>c_{0}>0$ and $B(u, u, t) \geq \int_{\Omega} u_{0} u d x$. then the solution $(u, c)$ of problem $(P)$ is global. ii) if $c>c_{0}>0$ and $B(u, u, t) \geq \int_{\Omega} u_{0} u d x$. then the solution $(u, c)$ of problem $(P)$ is global. Furthermore there exists $\sigma>0$ such that $\|u\|_{L^{2}(\Omega)} \leq e^{\sigma t}\left\|u_{0}\right\|_{L^{2}(\Omega)}$.

Proof. we put

$$
Z(t)=\frac{1}{2} \int_{\Omega} u^{2} d x
$$

we derivate the equation 2.26 and we use first equations of $\left(P_{1}\right)$ and $\left(P_{2}\right)$ to find

i) we have

$$
\frac{d Z}{d t}=\int_{\Omega} u_{0} u d x-B(u, u, t) \leq 0
$$

therefore

$$
Z(t) \leq Z(0)
$$

ii) we have

$$
\begin{aligned}
\frac{d Z}{d t}=\int_{\Omega} u_{0} u d x-B(u, u, t) & =\int_{\Omega} u_{0} u d x+\int_{\Omega} \nabla u^{2} d x-\int_{\Omega}(\delta \tau c+1) u^{2} d x+\delta \int_{\Omega} u \nabla u \nabla u_{0} d x \\
& =\int_{\Omega} u_{0} u d x+\int_{\Omega}\left(1+\frac{\delta \nabla u_{0}}{2}\right) \nabla u^{2} d x-\int_{\Omega}(\delta \tau c+1) u^{2} d x \\
& \leq\left|\delta \tau c_{0}+1\right|\|u\|_{L^{2}(\Omega)}^{2}=\sigma Z(t) .
\end{aligned}
$$

This implies that 


$$
Z(t) \leq Z(0) e^{\sigma t}
$$

Proposition 2.2. [16] Let $u_{0} \in L^{2}(\Omega)$ and $u \in C\left([0, T] ; L^{2}(\Omega) \cap L^{2}\left([0, T] ; H_{0}^{1}(\Omega)\right)\right)$ is the unique weak positive solution of $\left(P_{1}\right)$. If $u_{0} \geq 0$ in $\Omega$, then $u \geq 0$ in $] 0, T[\times \Omega$.

Proof. If $u_{0} \geq 0$ on $\partial \Omega$. Therefore $u^{-}=\min (u, 0) \in L^{2}\left([0, T] ; H_{0}^{1}(\Omega)\right)$. We obtain for all $0 \leq t \leq T$

$$
\frac{1}{2} \frac{d}{d t} \int_{\Omega}\left(u^{-}\right)^{2} d x+\int_{\Omega} B\left(u^{-}, u^{-}, t\right) d x=\int_{\Omega} u_{0} u^{-} d x
$$

Using the Lemma 2.1 and integrating with respect to $t$ from 0 to $T$, we get

$$
\frac{1}{2} \frac{d}{d t} \int_{\Omega}\left(u^{-}\right)^{2} d x+\alpha \int_{0}^{T}\|u(s)\|_{H_{0}^{1}(\Omega)}^{2} d s \leq \frac{1}{2} \frac{d}{d t} \int_{\Omega}\left(u^{-}(0)\right)^{2} d x=0 .
$$

Since $u^{-}(0)=\left(u_{0}\right)^{-}=0$. So $u^{-}=0$.

Conflicts of Interest: The author(s) declare that there are no conflicts of interest regarding the publication of this paper.

\section{REFERENCES}

[1] H.A. Barnes, Thixotropy a review, J. Non-Newtonian Fluid Mech. 70 (1997), 1-33.

[2] H. El-Gendy, M. Alcoutlabi, M. Jemmett, M. Deo, J. Magda, R. Venkatesan, A. Montesi, The propagation of pressure in a gelled waxy oil pipeline as studied by particle imaging velocimetry, AIChE J. 58 (2012), 302-311.

[3] H. Freundlich, Thixotropy, Paris, 1935.

[4] C.F. Goodeve, A general theory of thixotropy and viscosity, Trans. Faraday Soc. 35 (1939), 342-358.

[5] C.F. Goodeve, G.W. Whitfield, The measurement of thixotropy in absolute units. Trans. Faraday Soc. 34 (1938), 511-520.

[6] T. Hillen, K. Painter, Global Existence for a Parabolic Chemotaxis Model with Prevention of Overcrowding, Adv. Appl. Math. 26 (2001), 280-301.

[7] C.Mesikh, A. Guesmia, and S.Saadi, Global Existence and Uniqueness of the Weak Solution in Keller Segel Model. Glob. J. Sci. Front. Res. F, 14 (2014), 46-55.

[8] F. Moore, The rheology of cereamic slips and bodies. Trans. Br. Ceramic Soc. 58 (1959), 470-484

[9] A. Mujumdar, A.N. Beris, A.B. Metzner, Transient phenomena in thixotropic systems, J. Non-Newtonian Fluid Mech. 102 (2002), 157-178.

[10] Q.D. Nguyen, D.V. Boger, Thixotropic behaviour of concentrated bauxite residue suspensions. Rheol. Acta, 24(4) (1985), 427-437.

[11] J.O. Sibree, The viscosity of emulsions. Part I. Trans. Faraday Soc. 26 (1930), 26-36.

[12] J.O. Sibree, The viscosity of emulsions. Part II. Trans. Faraday Soc. 27 (1931), 161-176.

[13] P.R. de Souza Mendes, Modeling the thixotropic behavior of structured fluids, J. Non-Newtonian Fluid Mech. 164 (2009), 66-75. 
[14] J. De Vicente, C. Berli, Aging, rejuvenation, and thixotropy in yielding magneto-rheological fluids. Rheol. Acta, 52 (2013), 467-483.

[15] X. Zhang, W. Li, X. Gong, Thixotropy of MR shear-thickening fluids. Smart materials and structures, 19(12) (2010), 125012.

[16] G. Allaire, Analyse numérique et optimisation. Éd. de l'Ecole Polytechnique, Paris, 2005.

[17] L.C. Evans, Partial Differential Equations. Graduate Studies in Mathematics, 2nd edn. American Mathematical Society, Providence, RI, 2010. 